\title{
Hypertrophic tuberculosis of the rectum
}

\author{
P. R. HAWLEY, H. R. I. WOLFE, AND J. M. FULLERTON \\ From the Research Department, St. Mark's Hospital, City Road, London
}

Tuberculosis of the gastrointestinal tract occurs either as a primary lesion in areas where milk is unpasteurized, or secondary to a focus of tuberculosis elsewhere in the body, most commonly in the lungs. Two main types are seen: the tuberculous ulcer and the much rarer hypertrophic lesion, which occurs predominantly in the ileocaecal region $(85 \%$ of Bockus's (1964) cases were at this site), less commonly in the colon, and rarely in the rectum. Three cases of hypertrophic rectal tuberculosis are presented.

\section{CASE REPORTS}

CASE 1 The patient was a Pakistani male aged 25 who was referred with a rectal stricture of unknown aetiology. He worked in a factory and had entered the United Kingdom in 1960. Eight months before his admission to the Hospital for Tropical Diseases in February 1965 he had an acute attack of diarrhoea which lasted only two days, passing five watery stools each day with blood and mucus. He had subsequently resumed a normal bowel habit, but recently developed rectal pain with again a little blood and mucus in the stools. There was no constitutional upset and no loss of weight.

In 1955 he had a cervical node biopsy which showed tuberculosis and he received treatment with drugs, including streptomycin, for one month.

On examination he was found to have puckered scars on the neck and upper part of the chest and small discrete glands were palpable in the neck and left axilla. The sigmoid colon was loaded and on digital examination of the rectum a soft stricture could be felt. Sigmoidoscopy showed normal rectal mucosa to $9 \mathrm{~cm}$. At this level there was a soft stricture $2 \mathrm{~cm}$ long, through which the $\frac{1}{2}$ inch sigmoidoscope would just pass, with cobbled, pale and oedematous mucosa but no ulceration. There was another similar stricture at $17 \mathrm{~cm}$. Biopsies were taken from the rectal strictures, scar tissue from the neck, and an axillary lymph node. Histology of the rectal tissue showed a severe chronic inflammatory reaction in the submucosa with occasional sarcoid granulomas, in one of which was a giant cell. Zeil-Neilsen staining for acid-fast bacilli was negative. The biopsies from the neck and axilla showed caseous tuberculosis with acid-fast bacilli. Culture from the scar tissue was positive but from the rectum both culture and guinea-pig inoculation were negative.

Other investigations included the Frei test (negative); a complement-fixation test for lymphogranuloma venereum which was less than $\frac{1}{8}$; a Mantoux test which was positive $(1 / 100,000)$. A chest radiograph showed no evidence of old or active pulmonary tuberculosis.

Barium enema showed a rigid rectum and distal sigmoid colon. The mucosa was irregular and there were small projections in the distal sigmoid colon suggesting ulceration. The rectosacral distance was increased. The rest of the colon and the whole of the upper gastrointestinal tract were normal.

The diagnosis was considered to be hyperplastic tuberculosis of the rectum, but as the patient was generally well antituberculous drugs were withheld in an attempt to confirm it. Further biopsies were taken from the rectum in June 1965 and on this occasion sarcoid granulomas with giant cells were more numerous and one acid-fast bacillus was found on special staining. Guinea-pig inoculation and culture were again negative. While these investigations were being carried out the patient developed a large firm lymph node in the right inguinal region. This was removed and showed tuberculosis on histological examination.

Antituberculous drug therapy was started with streptomycin and Pasinah. He was followed up and three months after the institution of therapy the rectum was completely normal on sigmoidoscopy. He has continued on Pasinah and when last seen in November 1966 was very well.

CASE 2 The patient was a man aged 63 who came to the Out-patient Department in February 1966. He complained of difficulty in micturition for five years and of an anal ulcer for five months. He had been seen in a special clinic four months previously with a non-specific urethral discharge and had been treated with streptomycin and sulphamethoxypyridazine. He gave a past history of gonorrhoea in 1958. He had lost over a stone in weight and had recently developed a cough.

On examination the abdomen was a little distended with some suprapubic dullness but the bladder was not palpable. Inspection of the anus showed an ulcer on the right side with a bluish edge and coarsely granulating base. On digital examination the prostate was not large but there was a little anterior induration in the rectum. Sigmoidoscopy to $18 \mathrm{~cm}$ showed no abnormality. A diagnosis of Crohn's disease of the anus and chronic retention of urine was made. A barium meal and follow through showed a large irreducible hiatus hernia, and an intravenous pyelogram a non-functioning left kidney. The right kidney was hydronephrotic with a very dilated 


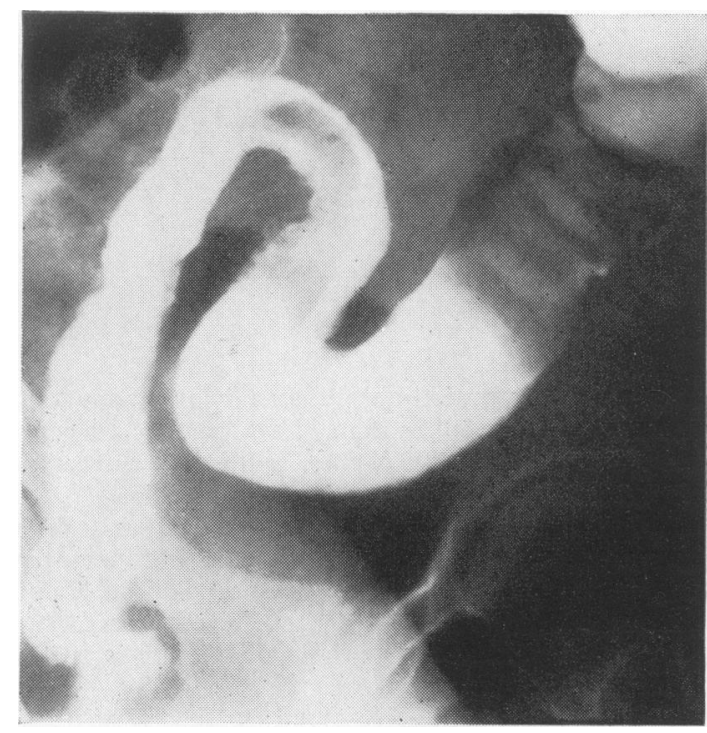

FIG 1a. Barium enema in case 1 showing non-distensible rectum with narrowing and slight mucosal ulceration.

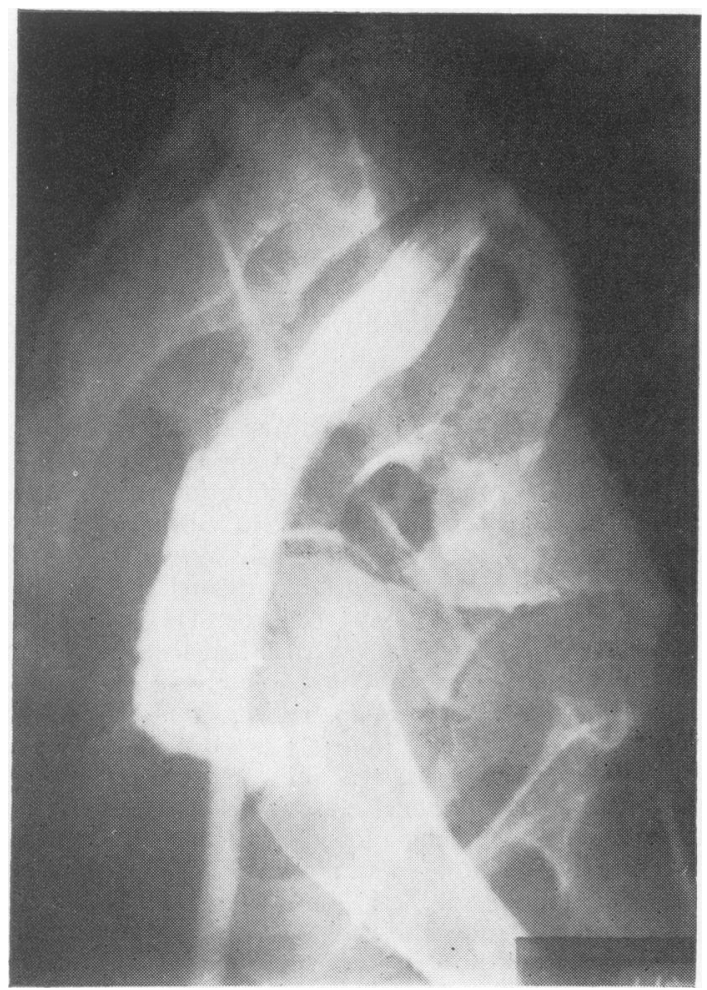

FIG. 1b. Lateral projection in case 1 showing increased recto-sacral distance. pelvis and, to a lesser extent, ureter. A biopsy of the anal ulcer showed epithelial granulomas and giant cells compatible with Crohn's disease.

In May he was admitted to hospital with a history of passing urine per rectum on defaecation four times a day. On examination he was wasted and anaemic with signs of consolidation in the right upper part of the chest. There was a suprapubic mass in the abdomen. The anal ulcer had enlarged and a second smaller one was present proximately. On digital examination there was an indurated mass in the rectum above the prostate. Sigmoidoscopy showed a large mass on the anterior wall above the prostate, with cobbled and ulcerated mucosa.

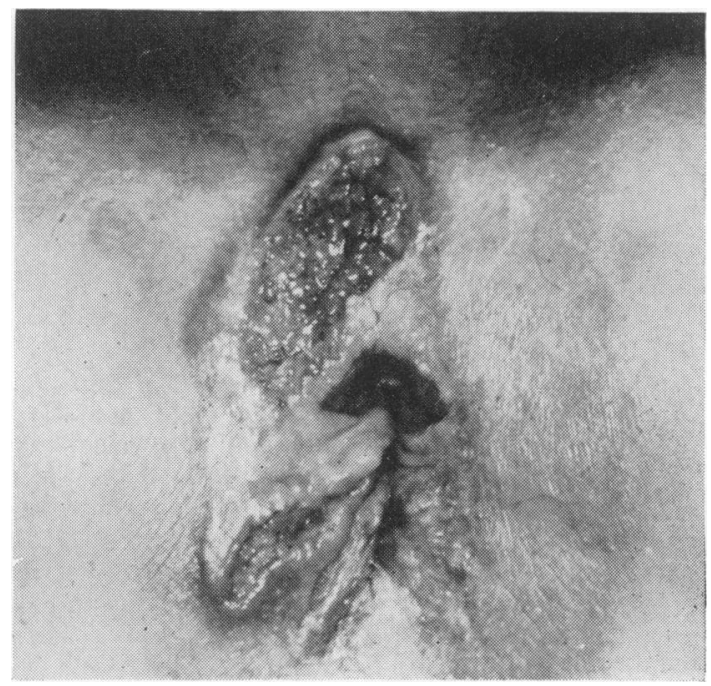

FIG. 2. Anal ulceration in case 2 .

Cystoscopy showed a fistula in the posterior wall of a trabeculated bladder just above the trigone, and a fibrosed bladder neck. The anal ulcer was again biopsied and on this occasion microscopy revealed caseating tuberculosis with acid-fast bacilli present. A biopsy of the rectal mass also showed tuberculosis with very little caseation but acid-fast bacilli were present.

Haemoglobin was $65 \%$, white blood count 8,800 and ESR $109 \mathrm{~cm}$ in one hour. A chest radiograph showed active tuberculosis with patchy consolidation in both upper and middle zones, more marked on the right than the left. A barium enema showed a filling defect in the rectum. Acid-fast bacilli were cultured from the sputum and on gastric lavage. Blood urea was $47 \mathrm{mg} / 100 \mathrm{ml}$. M.S.U. numerous pus cells, acid in reaction, and on culture a mixed growth. A specimen of urine tested for acid-fast bacilli was negative on culture.

Treatment was started by performing a laparotomy in May when the small and large bowel were found to be normal. There was a large mass in the rectum adherent to the bladder. A transverse colostomy was made and continuous catheter drainage of the bladder started.

Antituberculous drug therapy with streptomycin and 
Pasinah was begun. The patient was allergic to PAS and when the course of streptomycin had finished pyrazinamine and INAH were given and he has continued on this regime.

He rapidly improved and the sputum became negative for acid-fast bacilli in June. In November the rectal mass had resolved and on sigmoidoscopy the mucosa was completely normal. The bladder was opened at operation, and, the fistula being healed, a wedge resection of the bladder neck was performed. The right kidney and ureter had become almost normal in size and he is passing urine normally. The transverse colostomy was closed in February 1967.

CASE 3 The patient was a woman aged 66 who first attended hospital in June 1963, complaining of diarrhoea and pruritis ani which had been present for several months. There was no relevant past or family history.

On examination she was fairly healthy but had an inadequate personality. She was tender on palpation of the left colon. Rectal examination showed a chronic anterior and posterior fissure with surrounding inflammation and induration. Sigmoidoscopy showed a granular proctitis in the upper third of the rectum and lower sigmoid colon.

A chest radiograph showed healed foci of tuberculosis in both mid zones. A barium enema showed diverticular disease of the descending and sigmoid colon, and biopsy of the rectal mucosa chronic inflammatory changes only.

Treatment for the chronic fissures was refused. She was treated as a case of proctocolitis and attended the Out-patient Department from time to time.

In February 1964 she attended for treatment of the fissures in the anus, and histology of excised tissue showed chronic non-specific inflammatory changes only. Repeated sigmoidoscopy showed changes varying from mild granular proctitis to active inflammation with ulceration and bleeding. On four occasions she had to be admitted for treatment with Prednisone retention enemas of exacerbations of diarrhoea.

In December 1964 a further barium enema was done which showed a stricture of the sigmoid colon suggestive of malignant disease. The patient declined surgery at that time. In April 1965 she was readmitted to hospital with a recurrence of symptoms, anaemia, and mild heart failure. Three months later laparotomy was undertaken and this showed extensive diverticular disease of the descending and sigmoid colon. The upper rectum and rectosigmoid were thickened and nodular, suggesting Crohn's disease. An abdomino-perianal resection of the rectum was performed from which she made a good recovery.

The specimen consisted of the rectum and sigmoid colon $23 \mathrm{~cm}$ long. There was an ulcer at the anal canal at the site of the excised fissure, and $8.5 \mathrm{~cm}$ from the anal margin was a stricture $7 \mathrm{~cm}$ long with cobbled mucosa and superficial linear ulceration. The submucosa was $1 \mathrm{~cm}$ thick and tubercles were visible on the cut surface. The mucosa above the stricture was normal. Histology showed caseous tuberculosis with acid-fast bacilli.

Antituberculous therapy was instituted.

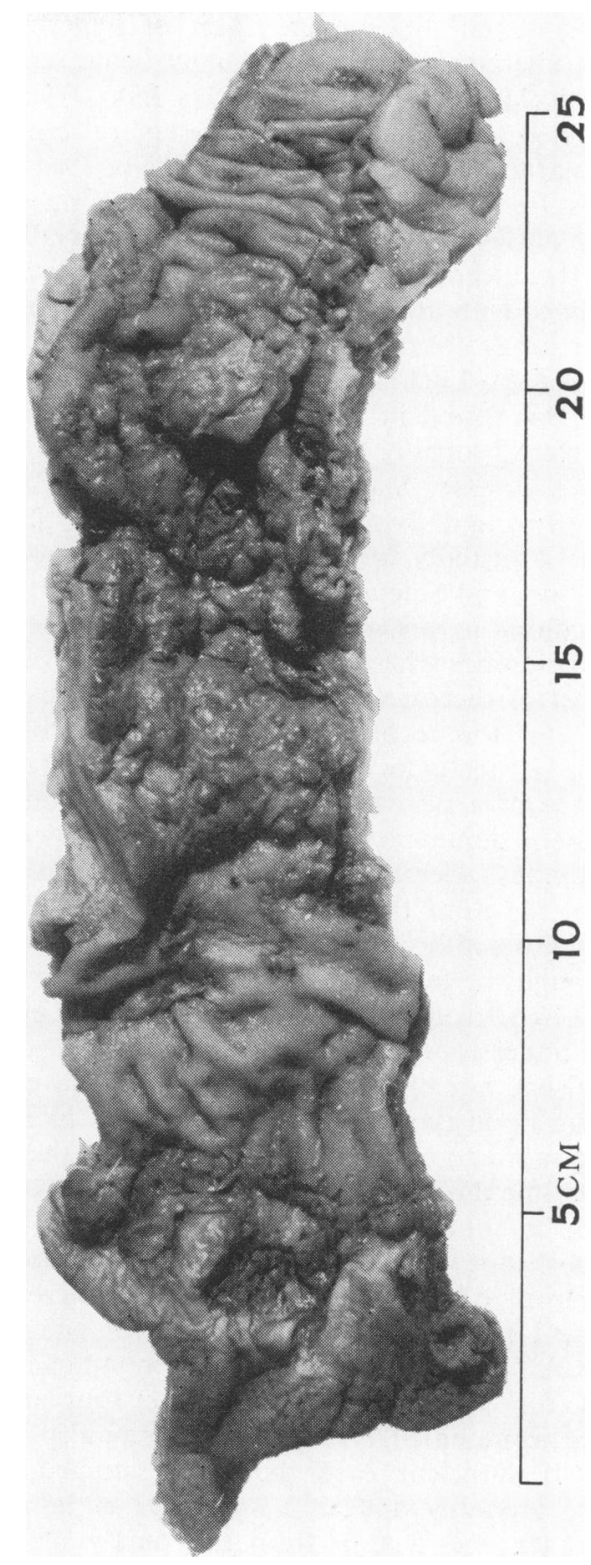

FIG. 3. Resected specimen showing hypertrophic tuberculosis of the rectum in case 3.

\section{DISCUSSION}

Hypertrophic tuberculosis of the gastrointestinal tract was first described by Hartman and Pilliet in 1891, who differentiated the lesion with grossly thickened submucosa and oedematous cobbled mucosa, with or without superficial ulceration, from that of a simple ulcer with an overhanging edge, and 
no submucosal thickening. Cases of hypertrophic rectal tuberculosis are rare, usually being reported as an isolated case (Davis, 1957; Jelks, 1939) or mentioned in discussion of the more common proximal lesion (Hancock, 1958; Recio, 1961).

It is frequently stated that hypertrophic tuberculosis of the colon is a primary condition as distinct from the ulcerative lesion which is almost always associated with an advanced primary lesion, usually in the lungs (Martin, 1932). Of a hundred cases described by Lockhart-Mummery (1923), no other lesion was found in 76 , but in retrospect many of these were probably due not to tuberculosis but to Crohn's disease. Davis (1957), Vogel (1952), and Pemberton and Brindley (1944) also state that the lesion is usually primary, although Rappaport, Burgoyne, and Smetana (1951) think that ileocaecal tuberculosis is rare without pulmonary tuberculosis in communities where milk is pasteurized. However, in most of the case reports of hypertrophic tuberculosis of the rectum, there is evidence that the disease is secondary. Each of our patients had evidence of a primary tuberculous focus; one had coexistent pulmonary tuberculosis, another cervical tuberculous adenitis with cutaneous tuberculosis, and in the third there was evidence of pulmonary tuberculosis although it seemed to be inactive.

Though the factors responsible for the formation of the hypertrophic lesion or the ulcerative are not fully understood, the site, the virulence of the organisms, and their number appear to have some bearing upon the problem. Tuberculous ulcers are found more commonly in the ileum than the colon or rectum; the hypertrophic lesion in the ileocaecal region or colon. If the acid-fast bacilli are few in number, not virulent, or partly attenuated, the hypertrophic lesion may result. It is of interest that in case 1 streptomycin was received for one month 10 years previously, and in case 2 streptomycin was given for a non-specific urethritis four months before he presented. A racial factor may also play a part. If sufficient cases could be studied, there would probably appear a spectrum of lesions in the colon and rectum from the purely ulcerative on one hand to the hypertrophic with no ulceration on the other. In case 1, where organisms were very few, there was no ulceration, but in cases 2 and 3 , where they were more numerous, ulceration was present.

The symptoms are usually not florid; there is a little diarrhoea with mucus in the stool and blood if the lesion is ulcerated: pain and tenesmus may be present. In addition there is often general malaise and loss of weight. In cases 1 and 3 there was slight diarrhoea, but in case 2 the only initial complaint was of an anal ulcer and dysuria. The speed at which the lesion can develop is indicated in case 2; only three months elapsed between the time the rectum was observed to be normal and the development of a tight stricture just above the prostate.

The local signs of the disease in the rectum may suggest the diagnosis, for the stricture is short, annular, and firm in consistency, a typically nodular surface and little ulceration. Sometimes further nodular areas of mucosa occur adjacent to the stricture, but above it the rectum and colon are normal. Unlike the ulcerative form of the disease in which local complications such as fissures and fistulas are fairly common, they do not seem to occur so readily in the hypertrophic type (LockhartMummery, 1923).

The diagnosis has to be made from carcinoma on the one hand, and other granulomatous strictures on the other. Of the latter, Crohn's disease is by far the most common and lymphogranuloma inguinale, actinomycosis, and possibly blastomycosis and coccidiomycosis are rarely seen. We believe that many of the cases previously described as due to hypertrophic tuberculosis were in fact due to Crohn's disease, a well-established entity in which isolated rectal and colonic lesions occur (Lockhart-Mummery and Morson, 1960). Crohn's disease is seen so often that it is easy to overlook the fact that the rare tuberculous lesion may be responsible for the stricture.

The differential diagnosis from carcinoma of the rectum is usually made without difficulty on microscopy of a biopsy specimen but it may be much more difficult to make a definite diagnosis of the much rarer malignant lymphoma. Mycotic disease can also be excluded histologically. However, if the biopsy shows a granuloma, the diagnosis cannot always be made on morphological grounds alone.

According to Gabriel (1963), lymphogranuloma inguinale was the most common cause of a benign stricture of the rectum, and he advised that it should always be excluded by a Frei test and a complementfixation test. He makes the point that the presence of tubercle bacilli does not exclude the possibility of the stricture being due to underlying lymphogranuloma, as this condition can sometimes be preceded or followed by tuberculosis or dysentery.

The most difficult differential diagnosis is between those conditions which histologically produce a tuberculoid reaction with epitheloid cells, namely, tuberculosis, Crohn's disease, and possibly sarcoidosis. In some reports in the past this difficulty has not been sufficiently appreciated (Vogel, 1952). The main reason for this is the paucity of tubercle bacilli (well illustrated by case 1). Stool culture is invariably negative. The culture and guinea-pig inoculation of biopsy material are often negative, 
and these tests are only of value if positive, and must be repeated if negative as the organisms are so scanty that negative results do not exclude tuberculosis (Davis, 1957). In the specimen, the epithelium is thickened by a mass of granulation tissue in submucosal and subserosal areas. Microscopy shows granulomas with many giant cells, usually without caseation, but acid-fast bacilli are very difficulty to find (Turell, Krakauer, and Maynard, 1953). However, even in the absence of caseation, conglomerate tubercles are a conspicuous feature, while in Crohn's disease the granulomas are usually discrete. Thickening of the wall in intestinal tuberculosis is due primarily to large masses of conglomerate tubercles; in Crohn's disease submucosal oedema and fibrosis are responsible (Rappaport et al, 1951).

Some of these difficulties are reflected in views previously expressed. Taylor (1945) would go as far as to state that the histology is identical and that Crohn's disease should only be diagnosed when extensive search has failed to reveal acid-fast bacilli. Fansler and Potter (1933) say that microscopy is not subject to possible error but Sweany (1947) reports that it is unreliable on its own. He found that guinea-pig inoculation is negative in $69 \%$ of cases of hypertrophic tuberculosis of the ileocaecal region and colon.

In conclusion, rectal tuberculosis must be considered as a possible diagnosis in any granuloma of the rectum, particularly when there is evidence of tuberculosis elsewhere. This can be excluded if the Mantoux reaction is negative. Radiological examination of the rectum will show a stricture and sometimes superficial ulceration but the appearances can be identical with those produced by Crohn's disease. The diagnosis will depend upon the histology of the biopsy and be confirmed by finding acid-fast bacilli. Culture and guinea-pig inoculation may not be positive and the diagnosis must not be excluded on these grounds. If it is suspected, biopsy should be repeated in an effort to find acidfast bacilli. The treatment is conservative with antituberculous drugs, for it is quite remarkable how quickly the lesion responds and resolution occurs. There is no indication to remove the rectum provided a definite diagnosis can be reached, but case 3 illustrates the difficulty of this as two biopsies were reported as showing only chronic inflammatory change. In cases 1 and 2 the rectal lesion healed quickly on adequate drug therapy and there was no residual stenosis.

We would like to thank Mr Norman Mackie, of the Department of Photography, at St. Mark's Hospital for the photographs.

\section{REFERENCES}

Bockus, H. L. (1964). Gastroenterology, 2nd ed., vol. 2, p. 328 Saunders, Philadelphia and London.

Davis, J. W. (1957). Hyperplastic tuberculosis of the rectum. Amer. J. Surg., 93, 490-492.

Fansler, W. A., and Potter, C. K. (1933). The surgical treatment of rectal tuberculosis. Surg. Gynec. Obstet., 57, 115-118.

Gabriel, W. B. (1963). Principles and Practice of Rectal Surgery, 5th ed., p. 423. Lewis, London.

Hancock, D. M. (1958). Hyperplastic tuberculosis of the distal colon. Brit. J. Surg., 46, 63-68.

Hartman, H., and Pilliet, A. H. (1891). Note sur une variété de typhlite tuberculeuse simulant les Cancers de la région. Bull. Mém. Soc. anat. Paris, 66, 471.

Jelks, J. L. (1939). Tuberculosis of the rectum. Trans. Amer. proctol. Soc., 40, 141-145.

Lockhart-Mummery, J. P. (1923). Diseases of the Rectum and Colon. Baillière, London. W. Wood, Baltimore.

Lockhart-Mummery, H. E. and Morson, B. C. (1960). Crohn's disease (regional enteritis) of the large intestine and its distinction from ulcerative colitis. Gut, 1, 87-105.

Martin, C. L. (1932). Ulcers of the rectum and sigmoid. Differentiation of tuberculous ulcers from amebic ulcers and chronic ulcerative colitis. J. Amer. med. Ass., 98, 27-31.

Rappaport, H., Burgoyne, F. H., and Smetana, H. F. (1951). The pathology of regional enteritis. Milit. Surg., 109, 463-502.

Pemberton, J. deJ., and Brindley, G. V., Jr. (1944). Tuberculosis of the rectum: report of case. Proc. Mayo Clin., 19, 46-50.

Recio, P. M. (1961). Tuberculosis of the large bowel. Dis. Colon Rect., 4, 439-441.

Sweany, H. C. (1947). Streptomycin in tuberculous enteritis. Amer. Rev. Tuberc., 56, 415-417.

Taylor, A. W. (1945). Chronic hypertrophic ileocaecal tuberculosis and its relation to regional ileitis (Crohn's disease). Brit. J. Surg., 33, 178-181.

Turell, R., Krakauer, J. S., and Maynard, A. de L. (1953). Colonic and anorectal function and disease. Int. Abstr. Surg., 96, 417-449.

Vogel, F. (1952). Hyperplastic tuberculosis of the rectum. Rev. Gastroent., 19, 733-738. 